\section{OC 8713 MAXIMISING RESEARCH IMPACT TO STRENGTHEN PUBLIC HEALTHCARE}

${ }^{1}$ Wilber Sabiitii ${ }^{*}{ }^{2}$ Nyanda E Ntinginya, ${ }^{4}$ Elizabeth Fb Msoka, ${ }^{3}$ Fred Orina, ${ }^{5}$ Ivan Mwebaza, ${ }^{2}$ Simeon Mwanyonga, ${ }^{3}$ Barbara Miheso, ${ }^{2}$ Erica Sanga, ${ }^{4}$ Aliphonce Liyoyo, ${ }^{3}$ Helen Meme, ${ }^{4}$ Blandina T Mmbaga, ${ }^{1}$ Ewan Chirnside, ${ }^{5}$ Moses Joloba, ${ }^{3}$ Evans Amukoye, ${ }^{5}$ Alphonse Okwera, ${ }^{6}$ Gibson S Kibiki, ${ }^{1}$ Stephen H Gillespie, ${ }^{5}$ Wilber Mwebaza, ${ }^{4}$ Nyanda E Msoka. 'University of St Andrews, Edinbrugh, UK; ${ }^{2}$ NIMR-Mbeya Medical Research Centre, Dar es Salam, Tanzania; ${ }^{3}$ Kenya Medical Research Institute, Nairobi, Kenya; ${ }^{4}$ Kilimanjaro Clinical Research Institute, Tanzania; ${ }^{5}$ Makerere University, Kampala, Uganda; ${ }^{6}$ East African Health Research Commission, Bujumbura, Burundi

10.1136/bmjgh-2019-EDC.38

Background Billions of dollars are spent on research globally every year, yet little is translated to public use through policy and/or commercialisation. For the few research findings that make it to policy, evidence in most LMICs shows they hardly see the light of implementation. Our EDCTP-funded TWENDE consortium used implementation of tuberculosis (TB) molecular diagnostics as a model to investigate the barriers, and opportunities to unlock them in order to maximise uptake of health research innovations into policy and practice. Methods Mixed methods approach including surveys, audits, in-depth interviews and focus group discussions (FGDs), policymaker dialogues to unravel the bottlenecks and how to overcome them.

Results 1119 respondents representing from Uganda, Kenya and Tanzania participated in the study. 19\% were district/ county health officers, $12 \%$ healthcare audits, $58 \%$ one-onone interviews and FGDs with healthcare practitioners, community leaders, TB patients and survivors, and care givers, and $11 \%$ policymaker workshops. Barriers: government poverty, family/individual poverty, incompatibility of technologies to existing infrastructure, low awareness and socio-cultural beliefs in the community were found. Stigma at community and healthcare levels was rife. Consequently, TB diagnostics were underimplemented and underutilised. Xpert MTB/RIF test was fully utilised in $\sim 10 \%$ of healthcare facilities (conducting 8 tests per day) whilst Line probe assay was implemented in less than $1 \%$ of the facilities.

Conclusion Based on our findings, we believe overcoming the barriers presents the opportunity to maximise research impact of public healthcare. This could be achieved through sustained public and practitioners' sensitisation to remove stigma to increase demand and utilisation of services; early interaction of researchers and policymakers to increase sense of ownership and acceptability of research innovations; early communication between developers and end-users to align the tools with the needs and existing infrastructure capacity; and increased affordability of innovations through socioeconomic empowerment programmes.

\section{OC 8715 GOING FURTHER TOGETHER: THE ROLE OF THE EU IN SUPPORTING REGULATORY HARMONISATION OF HEALTH TOOLS IN AFRICA}

Agustin Martin Lasanta. DSW Brussels [Deutsche Stiftung Weltbevölkerung], Belgium

\subsection{6/bmjgh-2019-EDC.39}

Background On May 20, 2018, African governments adopted the Treaty for the establishment of the African Medicines Agency (AMA) as a stepping stone in the strengthening and harmonising of medical products regulation on the continent.
Many countries in Africa lack robust and comprehensive regulatory systems supporting the research and registration of health products, hampering the translation of health research and innovation into public health impacts. The AMA could substantially improve the access to quality-assured, safe and effective health solutions and technologies in Africa and around the world. As the main trade partner and donor to Africa, the European Union (EU) has a key role to play in supporting this effort.

Method Desk research and case-study analyses of key initiatives to strengthen medicines regulation in Africa were conducted. Specifically, the EDCTP, the African Vaccine Regulatory Forum (AVAREF), the African Medicines Regulatory Harmonisation Programme (AMRH) and the Zazibona Process; to assess the gaps, needs and opportunities for EU collaboration using actual and reconfigured EU-Africa funding and policy cooperation mechanisms.

Results and conclusions This presentation will explore initiatives aimed at regulatory strengthening and harmonisation in Africa and propose policy recommendations on how to improve EU efforts in order to support these initiatives in a more systematic and synergetic manner. The recommendations fall into four categories: EU-Africa priority-setting, coordination, funding and capacity-building actions. Recommendations explore, for instance, advancing the national implementation of the African Union Model Law on Medical Products Regulation by all African countries, enlarging the scope of EDCTP regulatory strengthening actions to include Africa's Regional Centres of Regulatory Excellence (RCOREs) or improving the attractiveness and incentive system of the EMA's article 58 procedure for African governments, researchers and manufacturers.

\section{OC 8716 ENHANCING RESEARCH ETHICS REVIEW CAPACITY IN SUDAN}

${ }^{1}$ Shaza Abass*, ${ }^{2}$ Sara-Lavinia Brair, ${ }^{3}$ Shahd Osman, ${ }^{4}$ Henry Silverman. ${ }^{1}$ University of Khartoum, Sudan; ${ }^{2} \mathrm{Al}$ - Neelain University, Khartoum, Sudan; ${ }^{3}$ Public Health Institute, Khartoum, Sudan; ${ }^{4}$ University of Maryland, USA

\subsection{6/bmjgh-2019-EDC.40}

Background In Sudan, there is an increase in health research in a situation of scarce resources and limited counteractive quality assurance in research ethics. The aim of this project was to enhance the ethical review system in Sudan.

Methods Our framework for enhancing the ethical review capacity was based on the context of Sudan with emphasis on governance, coordination, feasibility, efficiency and sustainability. Activities conducted to achieve our goals included reviewing the guidelines that govern human subjects research, enhancing the governance of national authorities (National Health Research Ethics Committee and National Medicine and Poisons Board), improving coordination between the national authorities by developing a consensus clarifying their roles and functions, capacity building for the oversight bodies and institutional ethical review committees (RECs) as well as establishing a network of research ethics committees.

Results The guidelines that govern human subjects research in Sudan were reviewed and updated. In addition, a consensus document was endorsed to clarify the roles of the national regulatory authorities creating channels of coordination and cooperation between them and institutional RECs. Thirty-nine RECs from different parts of Sudan have been trained and the results of the pre/post test have shown an increase in the 
knowledge score among trainees $(\mathrm{p}<0.05)$. The project has also provided a platform for sharing experiences and maintaining partnerships with regional and international institutes in addition to provision of technical support for newly established RECs.

Conclusion We believe that the array of activities conducted through this project had enhanced the governance, coordination, feasibility and, efficiency of the ethical review system in Sudan.

\section{OC 8717 TRANSFER OF LEADERSHIP, WHAT DO WE NEED?}

Stellah Mpagama. Kibong'oto Infectious Diseases Hospital, Kilimanjaro, Tanzania

\subsection{6/bmjgh-2019-EDC.41}

Background The Pan African Consortium for the Evaluation of Anti-Tuberculosis Antibiotics (PanACEA) was designed to build clinical tuberculosis (TB) trial capacity whilst conducting clinical trials investigating novel and existing agents to shorten and simplify TB treatment. One of the objectives of the programme is to foster leadership development in sub-Saharan TB-endemic countries to move leadership to African partners in the PanACEA research programmes.

Methods and results In PanACEA 1 the participation of the sites on the consortium board was important to foster leadership development. African investigators now make up a large part of the consortium leadership and are actively developing new concepts. Delegates of the sites visited the annual PanACEA General Assembly meetings, where they could gain knowledge, actively participate in the meetings and discussions and network with others from the TB research community. Various sites participated at TB research community conferences (e.g. CROI, Lung Health Meeting) where PanACEA members gave presentations and could collaborate with other TB trial networks.

In PanACEA 2 all clinical trials are co-led by a European established researcher and an African Principal Investigator (PI), to ensure that African scientists are trained and mentored to lead in every aspect essential to clinical trial delivery, from trial and data management to statistical analysis and trial design, and from financial management to laboratory science. The capacity development cores, which serve as operational support for all PanACEA studies and provide high level oversight, also use the European and African countersystem, including senior and junior co-chairs among the African trial sites.

An example of leadership transfer is a large EDCTP application for the development of a new compound in MDR TB (FACE-MDR-TB) in which Stellah Mpagama is the lead applicant.

Conclusion The PanACEA consortium has actively facilitated a transfer of leadership programme which may be successful in future consortia.

\section{OC 8718 WHAT DID WE LEARN FROM PANACEA 1 CLINICAL TRIALS?}

Nyanda E Ntinginya. NIMR - Mbeya Medical Research Centre, Tanzania

10.1136/bmjgh-2019-EDC.42

Background The Pan African Consortium for the Evaluation of Anti-Tuberculosis Antibiotics (PanACEA) was designed to build clinical tuberculosis (TB) trial capacity whilst conducting clinical trials on novel and existing agents to shorten and simplify TB treatment. One of the objectives was to conduct, mentor and monitor observational and clinical studies at sites in 6 Sub-Saharan TB-endemic countries (Gabon, Kenya, South Africa, Tanzania, Uganda and Zambia)

Methods Learning through experience. All centres in the 6 countries self-assessed their requirements for capacity development in the following fields: a) clinical staff availability and experience; b) TB laboratory infrastructure and staff; c) safety laboratory infrastructure and staff; d) clinical site facilities and equipment; e) pharmacy facilities and staff; IT facilities; and f) overall training needs of site personnel.

Results From March 2011 - June 2014, we conducted four epidemiological studies (characterising TB patient populations in preparation for future studies) and five phase II studies (GCP standard intervention trials).

By working together in epidemiological and clinical trials, the sites identified their needs for resources and training as well as developing capabilities to perform independent largescale TB clinical trials beyond PanACEA-initiated trials. Through the ReMoxTB study, for example, laboratories were brought to an international standard for safety and mycobacterial expertise. Furthermore, through developing skill-sets related to EBA studies, sites have since then attracted other sponsors for further studies.

Sites could be mentored to perform GCP-compliant clinical TB trials that is built on sound physical infrastructure, training and strong on-site leadership.

Conclusion The learning-by-doing approach meant that staff could be trained whilst acquiring new core competencies and revealing operational gaps. Our experience of conducting TB trials within an environment of mentoring, networking and training has provided a platform for establishing future sustainable research centres that has capacities to conduct highly regulated studies.

\section{OC 8721 WANECAM II - A CLINICAL TRIAL PROGRAMME TO ASSESS SAFETY, EFFICACY AND TRANSMISSION- BLOCKING PROPERTIES OF A NEW ANTIMALARIAL KAF156 (GANAPLACIDE) IN UNCOMPLICATED MALARIA IN WEST AND CENTRAL AFRICA}

${ }^{1}$ Abdoulaye Djimde ${ }^{*}$, on behalf of the WANECAM II consortium, ${ }^{6}$ Martin P Grobusch, ${ }^{4}$ Rella Zoleko Manego, ${ }^{4}$ Ghyslain Mombo-Ngoma, ${ }^{7}$ Stephane Picot, ${ }^{1}$ Issaka Sagara, ${ }^{8}$ Colin Sutherland, ${ }^{1}$ Aminatou Kone, ${ }^{1}$ Ogobara K Doumbot, ${ }^{9}$ Jose Pedro Gil, ${ }^{9}$ Anders Björkman, ${ }^{12}$ Steffen Borrmann, ${ }^{3}$ Issiaka Soulama, ${ }^{1}$ Bakary Fofana, ${ }^{11}$ Stephan Duparc, ${ }^{1}$ Alassane Dicko, ${ }^{10}$ David Hughes, ${ }^{10}$ Cornelis Winnips, ${ }^{2}$ Sodiomon B Sirima, ${ }^{5}$ Eric Adehossi, ${ }^{13}$ Jean-Bosco Ouedraogo, 'Laurent Dembele, ${ }^{3}$ Issaka Zongo, ${ }^{11}$ Sophie Biguenet, ${ }^{2}$ Edithe Ilboudo-Sanogo, ${ }^{3}$ Aminata Fofana. ${ }^{1}$ Université des Sciences, des Techniques et des Technologies de Bamako (USTTB), Mali; ${ }^{2}$ Groupe de Recherche Action en Santé (GRAS), Burkina Faso; ${ }^{3}$ Institut des Sciences et Techniques (INSTech), Ouagadougou Burkina Faso; ${ }^{4}$ Centre de Recherches Médicales en Lambaréné (CERMEL), Libreville, Gabon; ${ }^{5}$ University Abdou Moumouni of Niamey, Niger; ${ }^{6}$ Academic Medical Center (AMC), Amsterdam, The Netherlands; ${ }^{7}$ University Lyon 1, France; ${ }^{8}$ London School of Hygiene and Tropical Medicine, UK; ${ }^{9}$ Karolinska Institute (KI), Stockholm, Sweden; ${ }^{10}$ Novartis Pharma $A G$, Basel, Switzerland; " ${ }^{11}$ Medicines for Malaria Venture (MMV), Geneva, Switzerland; ${ }^{12}$ Eberhard Karls Universität Tübingen, Germany

\subsection{6/bmjgh-2019-EDC.43}

Background Despite major progress in the past decade, malaria remains a major public health problem in sub-Saharan Africa. West and Central Africa account for nearly $2 / 3$ of the burden currently attributable to falciparum malaria. Artemisinin-based 